\title{
Optimisation of mixing performance of helical ribbon mixers for high throughput applications using computational fluid dynamics
}

DOI:

10.1016/j.cherd.2018.01.053

\section{Document Version}

Accepted author manuscript

Link to publication record in Manchester Research Explorer

Citation for published version (APA):

Mihailova, O., Mothersdale, T., Rodgers, T., Ren, Z., Watson, S., Lister, V., \& Kowalski, A. (2018). Optimisation of mixing performance of helical ribbon mixers for high throughput applications using computational fluid dynamics. Chemical Engineering Research and Design. https://doi.org/10.1016/j.cherd.2018.01.053

Published in:

Chemical Engineering Research and Design

\section{Citing this paper}

Please note that where the full-text provided on Manchester Research Explorer is the Author Accepted Manuscript or Proof version this may differ from the final Published version. If citing, it is advised that you check and use the publisher's definitive version.

\section{General rights}

Copyright and moral rights for the publications made accessible in the Research Explorer are retained by the authors and/or other copyright owners and it is a condition of accessing publications that users recognise and abide by the legal requirements associated with these rights.

\section{Takedown policy}

If you believe that this document breaches copyright please refer to the University of Manchester's Takedown Procedures [http://man.ac.uk/04Y6Bo] or contact uml.scholarlycommunications@manchester.ac.uk providing relevant details, so we can investigate your claim.

\section{OPEN ACCESS}


${ }^{1}$ Unilever Research and Development, Quarry Road East, Port Sunlight, CH62 4ZD, United

$7 \quad$ Kingdom

$8{ }^{2}$ School of Chemical Engineering and Analytical Science, The University of Manchester,

9 Oxford road, Manchester, M13 9PL, United Kingdom

${ }^{3}$ Siemens PLM Software, 200 Shepherds Bush Road, W6 7NL, United Kingdom

*Corresponding author: olga.mihailova@unilever.com

- 98 Helical mixer designs were screened using STAR-CCM+ and HEEDS package

- Experimental validation was performed using ERT and torque measurements 
19 The work presented focuses on the optimisation in a $1 \mathrm{~L}$ vessel using an anchor with a helical

20 ribbon design using CFD and a learning algorithm, optimised by minimisation of the torque

21 output of the mixer and the homogeneity of the mixture in the vessel after a defined mixing

22 time. The results were successfully validated experimentally using Electrical Resistance

23 Tomography (ERT) and direct torque measurements.

24 The study determined that the height of the mixer is a key factor in the performance of the

25 mixer, with other significant factors present, but with a lower impact. For the case of

26 torque, all design features of the mixer which increase the size, i.e. surface area acting

27 against motion, were found to be significant in increasing the modelled torque response.

28 The Auger screw was found to have no significant impact on either mixing and torque

29 response.

30 The results illustrate the capability of optimisation algorithms to achieve results comparable

31 to those achieved experimentally, while assessing a significantly larger number of design

32 options and optimising for several performance indicators simultaneously. 
35 Due to several factors mixing becomes more difficult as the equipment is scaled down, therefore traditional mixer geometries might prove inefficient in a smaller vessel. However,

37 achieving efficient mixing in smaller vessels is highly desirable, especially for highthroughput automated systems used for research and development. Such systems are expected to produce high quality outputs at scales as small as $100 \mathrm{~mL}$, while formulating products with complex rheological properties, unlocking research and development capability and integrating such capabilities into automated high-throughput formulation platforms.

An example of such high throughput device is the Geoff formulation robot (LabMan, UK), located at the Materials Innovation Factory at the University of Liverpool. This automated formulator is designed to produce up to 20 distinct $1 \mathrm{~L}$ formulations in a 24 -hour window. However, as the formulator targets customers from across the FMCG industry, it is often tasked with processing complex non-Newtonian fluids (Balzer et al., 1995). The standard agitation geometry on the robotic formulator includes a single stage pitched blade turbine, a 2-arm anchor agitator and a high shear device. This geometry can find it challenging to effectively blend more viscous and complex fluids, reducing the throughput capability of the platform (Ameur, 2016), and was therefore targeted for improvement using a range of

\section{2 techniques.}

53 The application of helical ribbon mixers to enhance the bulk mixing of non-Newtonian fluids in cylindrical vessels is well known and widely researched, with the various designs being the recommended solution for achieving high homogeneity in such systems (Ayazi Shamlou and Edwards, 1985; Brito-De La Fuente et al., 1997; Doraiswamy et al., 1994; Gijón- 
57 Arreortúa and Tecante, 2015; Zhang et al., 2008). Therefore, helical mixers were chosen as

the lead option for improving mixing on the small scale high throughput formulator platform, with the aim to improve the mixing performance of the anchor assembly, while keeping the geometry relatively simple, to ensure effective cleaning using the automated cleaning protocols on the robot. However, due to a number of design constraints resulting from the integration of the mixer into an existing platform a standard mixer design could not be implemented and therefore an optimisation study was performed, where the maximising the homogeneity of the final mixture after a given mixing time and minimising the torque response of the mixer were the optimisation parameters.

Computational fluid dynamics (CFD) is a powerful tool in mixing research, as it allows to assess and compare the performance of various mixer geometries without the need for experimentation (Han et al., 2012; Vicum et al., 2004). CFD provides a further benefit of tracking the evolution of the process across the volume of the vessel, which is often not practical when studying real industrial systems experimentally, due to the impermeable nature of the construction materials of the vessels and often the fluids within. In addition, it is possible to couple a CFD modelling tool with an optimisation tool, where a target can be set, for example to minimise energy consumption of the mixer, and some features of the design can be varied by the optimiser to achieve the set goal (Hanada et al., 2016; Palacz et al., 2016). For the purposes of this study Siemens STAR-CCM+ CFD package and HEEDS optimisation package were used. However, to ensure the quality of the CFD predictions, it is advisable to carry out experimental validation on select cases, to ensure model compliance. Mixing is often difficult to validate, due to the challenges posed by the equipment, as discusses previously. Nevertheless, a number of techniques exist which allow process 


\section{Materials and Methods} and consists of 3 components which promote mixing: propeller turbine, high shear mixer

tracking by measuring some form of tracker or difference between the materials being blended, such as planar laser image fluorescence (PLIF) (Ramsay et al., 2016), Particle Tracking Velocimetry (PTV) (Alberini et al., 2017), Positron Emission Particle Tracking (PEPT) (Mihailova et al., 2015), Magnetic Resonance Imaging (MRI) (McCarthy et al., 2002) and Electrical Resistance Tomography (ERT) (Rodgers and Kowalski, 2010), which used for validation purposes in this work.

3D printing has become a mature technology which is used across industries for the rapid manufacture of both prototypes for illustrative purposes and functional parts in a range of materials (Chua et al., n.d.). The validation work for this project was made possible through rapid prototyping using 3D printing, in particular selective laser sintering (SLS), which allowed the production of the complex mixer geometries in-house, providing the opportunity to test and evaluate the new designs quickly.

The combination of the abovementioned techniques allowed to design, analyse and validate a wide range of mixer design alternatives to improve the mixing performance of the highthroughput formulator platform. Moreover, the CFD and the rapid prototyping approaches were found to be exceptionally complimentary, as the models generated using CFD could be used directly to manufacture prototypes for experimental validation.

\subsection{Starting geometry and testing platform}

The robotic platform mixer assembly is held on the lid of the individual processing stations and an anchor-scraper, a typical example of which is shown in Figure 1a. Further to the 
mixer elements, there are dip in temperature and $\mathrm{pH}$ sensors are present on the module lid.

103 This mixer assembly was designed with a wide range of product rheologies and processing

104 requirements in mind, while still allowing the geometry to be easily cleaned in between 105 investigated formulations.

106 For the purposes of optimisation of the robotic formulator through formulation cycle time

107 reduction as well as to improve the mixing performance in more challenging formulations

108 (e.g., high viscosity and non-Newtonian liquids) several modifications have been

109 investigated, predominately aimed at optimisation of turbine and anchor geometries. For

110 the purposes of this study the focus is solely on the modifications to the anchor-scraper,

111 where the inspiration for the modifications of the simple two arm anchor has come from

112 the popular helical ribbon mixers which have been shown to provide enhanced mixing in

113 fluids exhibiting a wide range of rheological properties.

114 All design modifications are assessed using the purpose built stand-alone mixing unit, which

115 replicates all the design features of the robotic mixer platform, but the speed and runtime

116 of the mixer is controlled manually, as opposed to via a pre-set programme. In addition, the

117 anchor shaft has been modified with a quick-release clasp, allowing to swap out mixer

118 geometries without interfering with the rest of the machine. Furthermore, the lid lowering

119 mechanism on the stand-alone rig ensured that the geometry is brought into the same

120 position with respect to the sample pot, with a consistent immersion level and orientation. 


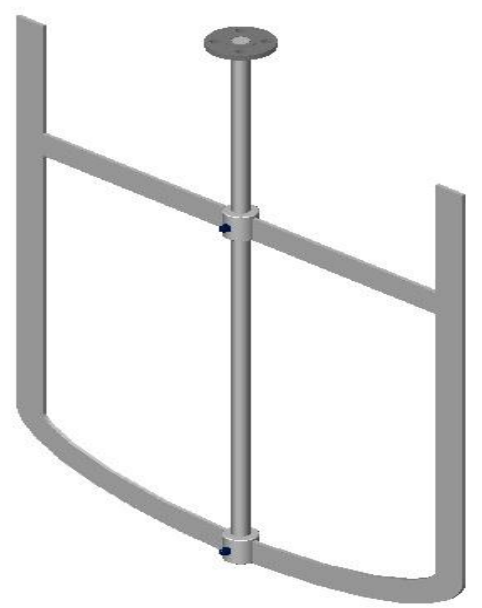

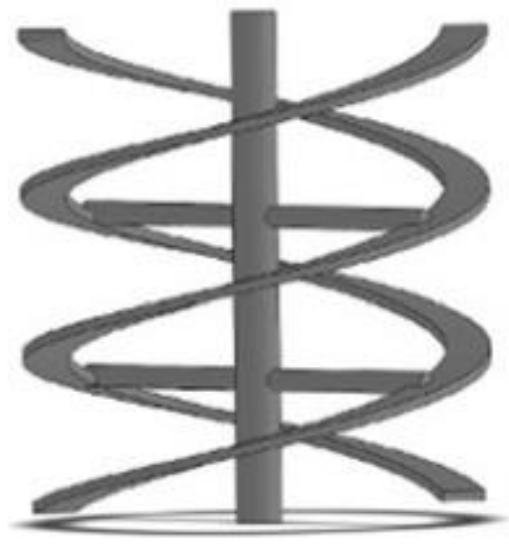

b
121

122 However, due to the presence of other elements in the mixer volume (i.e., temperature and

$123 \mathrm{pH}$ probes), it is not possible to accommodate the traditional central shaft ribbon support,

124 typical for helical ribbon mixers, as shown in Figure 1b. Therefore, the helical ribbons 125 differentiated by seven key design features: (1) height of the anchor/helix assembly, (2)

131 width of the helix, (3) thickness of the helix, (4) number of helix turns, (5) number of anchor arms, (6) height of the central auger screw, and (7) width of the central auger screw. The

133 number of anchor arms is varied to accommodate the increase in the number of helical

\subsection{Computational fluid dynamics (CFD) modelling}

Siemens STAR-CCM+ software was used to carry out the modelling of 98 mixer designs ribbon turns, as described previously. However, the two are independent variables, i.e., 
some of designs tested can have the same number of helical ribbon turns, but a different

136 number of anchor arms, or vice versa.

137 The simulation was set to assess the mixer performance in a fluid with the rheological 138 profile based on that of a typical commercially available shampoo formulation, typically 139 fitting a Carreau model, shown in Equation 1, where $\mu_{\text {eff }}$ is the apparent viscosity (Pa.s), $\mu_{0}$ is 140 the viscosity at low viscosity, i.e. the Newtonian plateau (Pa.s), $\mu_{\infty}$ is the viscosity at infinite 141 shear rate (Pa.s), $\dot{\gamma}$ is the shear rate $\left(\mathrm{s}^{-1}\right), n$ is the Power law index $(-)$ and $\lambda$ is the relaxation 142 time (s). The fluid has a distinct Newtonian plateau at lower shear rates, with the average 143 viscosity of 10 Pa.s, and a shear thinning region at higher shear rates, as shown in Figure 2 , 144 where the coefficients are as follows, $\mu_{0}=9.3$ Pa.s, $\mu_{\infty}=0$ Pa.s, $n=0.18$ and $\lambda=$ $1450.037 \mathrm{~s}$ Such rheological behaviour is typical of personal and household care products, as 146 well as some foods and other fast-moving consumer goods (FMCGs).

$$
\mu_{e f f}=\mu_{\infty}+\left(\mu_{0}-\mu_{\infty}\right)\left(1+(\lambda \dot{\gamma})^{2}\right)^{\frac{n-1}{2}}
$$

147 The fluid behaviour was based on the above model, by solving the Navier-Stokes equations

148 numerically using the widely used SIMPLE algorithm (Ferziger and Perić, 2002), assuming 149 laminar flow. 


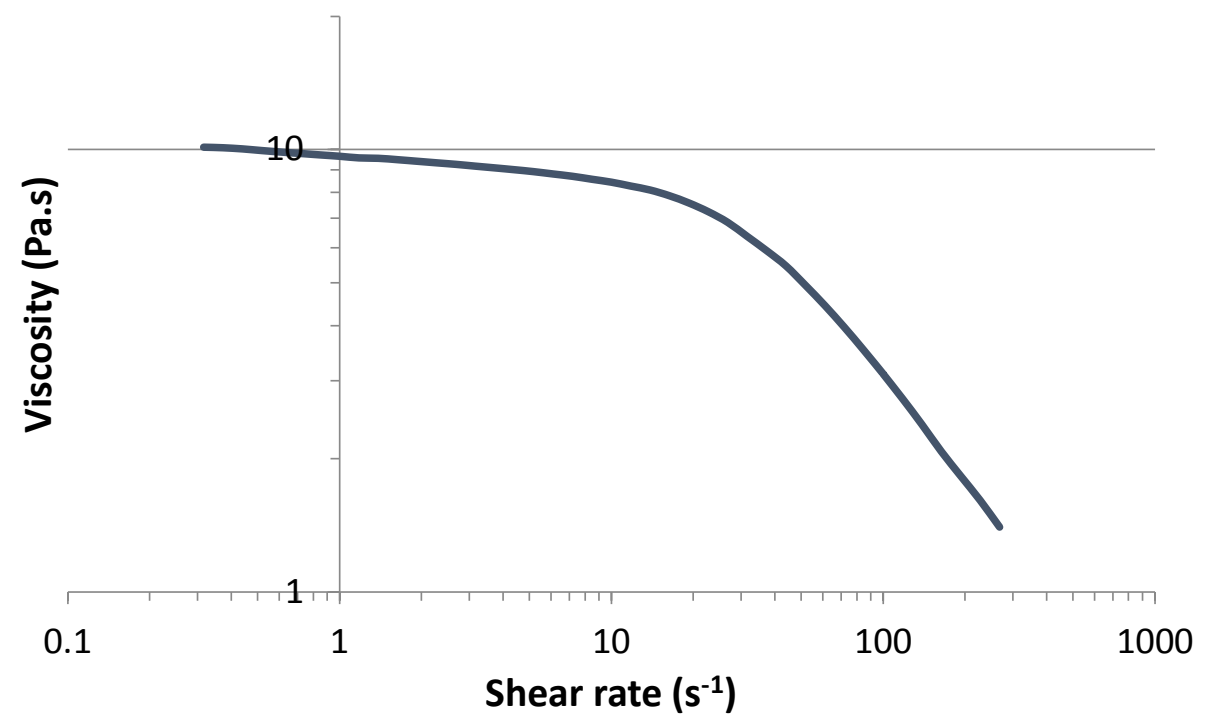

150

Figure 2. Flow curve of the fluid used in the CFD optimisation study

152 The simulation was set to run for a finite number of time steps, corresponding to 10 minutes

153 of the mixer running in real time, at the end of which the homogeneity of the system was

154 assessed. For these simulations, all the elements of the mixer assembly were present, to

155 allow for the assessment of any dead-zones caused by the presence of obstructions, such as

156 the $\mathrm{pH}$ meter. However, only the anchor assembly was providing the mixing duty, with the

157 high shear homogeniser and the turbine impeller present in the system, but static.

158 To assess the mixing performance of each anchor design tracers were introduced into the

159 simulation, where the tracer is modelled as a liquid with the same properties as the bulk,

160 but is tagged with a different concentration parameter, similar to injecting a liquid

161 containing a dye into the bulk. Homogeneity describes the distribution of this tracer in a

162 vessel volume, where if the tracer is distributed equally, the resulting number is 1 , while

163 homogeneity of less than 1 implies some local concentration gradients, with lower numbers

164 indicating an increasingly poorly mixed system, as calculated using Equation 2. 


$$
\text { Homogineity of } \phi=1-\frac{\sum_{c}\left|\phi_{c}-\bar{\phi}\right| V_{c}}{2|\bar{\phi}| \sum_{c} V_{c}}
$$

165 where $\bar{\phi}$ is the volume average of $\phi, \phi_{c}$ is the value for the selected scalar in a cell and $V_{c}$ is 166 the cell volume.

167 As part of the study the torque response of the mixer was also modelled and was used as a metric of the size of the geometry, it can be defined as the mass resistance to mixing, and 169 for the case of the same fluid used larger designs can be expected to exhibit higher 170 resistance and result in a higher torque response, due to the increase in the surface area 171 normal to the direction of motion of the anchor (Ameur, 2016; Kuhs et al., 2017). As the

172 final optimised design was due to be installed on an automated formulation platform which

173 is also self-cleaning using a spray down approach, it is crucial to keep the complexity of the 174 anchor-helix assembly low, as a bulkier design could be more challenging to clean and 175 additionally impede the cleaning of the instruments contained within the assembly (e.g., $\mathrm{pH}$ 176 meter or high shear mixer). Therefore, for this application it is desirable to keep the torque 177 low, while maximising the homogeneity of the system at the end of the mixing operation.

178 The torque response was calculated based on the forces exerted on the surfaces due to 179 shear and pressure in the system, where the total force on a surface is computed as follows

$$
f=\sum_{f}\left(\mathrm{~F}_{f}^{\text {pressure }}+\mathrm{F}_{f}^{\text {shear }}\right) \cdot \mathrm{n}_{f}
$$

180 Where $\mathrm{F}_{f}^{\text {pressure }}(\mathrm{N})$ and $\mathrm{F}_{f}^{\text {shear }}(\mathrm{N})$ are pressure and shear forces on the surface face, 181 respectively, and $f$ is the surface face and $\mathrm{n}_{f}$ are a user specified direction in which to 182 compute the force. 
183 The force that the fluid exerts on the surface, the pressure force vector $\left(\mathrm{F}_{f}^{\text {pressure }}\right)$, is 184 computed as follows

$$
\mathrm{F}_{f}^{\text {pressure }}=\left(p_{f}-p_{\text {ref }}\right) a_{f}
$$

185 Where $p_{f}$ is the face static pressure $(\mathrm{Pa})$ and $a_{f}$ is the face area vector $\left(\mathrm{m}^{2}\right)$

186 While the shear force exerted on the surface by the fluid, the shear force vector $\left(\mathrm{F}_{f}^{\text {shear }}\right)$

$187(\mathrm{~Pa})$, is computed as show in Equation 5.

$$
\mathrm{F}_{f}^{\text {shear }}=-T_{f} \cdot a_{f}
$$

Where $T_{f}$ is the stress tensor $(\mathrm{Pa})$ at face $f$

189 Torque can be further calculated when the forces on the individual mixer faces are known.

$$
\tau=r \cdot f
$$

190 Where $\tau$ is the torque (N.m) and $r$ is the distance of the element for which the force was 191 calculated from the shaft $(\mathrm{m})$, which is based on the distance of each mesh element from 192 the shaft.

193 An example of the polyhedral mesh used in the STAR-CCM+ is shown in Figure 3, where it 194 can be seen that the mesh is finer around the areas where the mixer is present, i.e. anchor 195 and central screw, to closely track the torque and mixing response. The mesh is coarser in 196 the bulk of the mixer, which allows to reduce the computational power required to 197 complete each simulation step. The average mesh count is 10 million cells, with a standard deviation of 2 million, which varies depending on the individual geometry of the of the 199 anchor assembly. The model is steady state with a frozen rotor, also known as the moving reference frame (MRF). 
201 After the flow field was converged based on asymptotic stopping criteria for the torque (+/-

$2020.01 \mathrm{Nm}$ ) and minimum criteria for the continuity residual the tracer was introduced by

203 solving the scalar transport equation incorporating diffusion, convection and

204 accumulation. Time was then advanced using a $1^{\text {st }}$ order implicit methodology using a time

205 step size of 0.005 s.

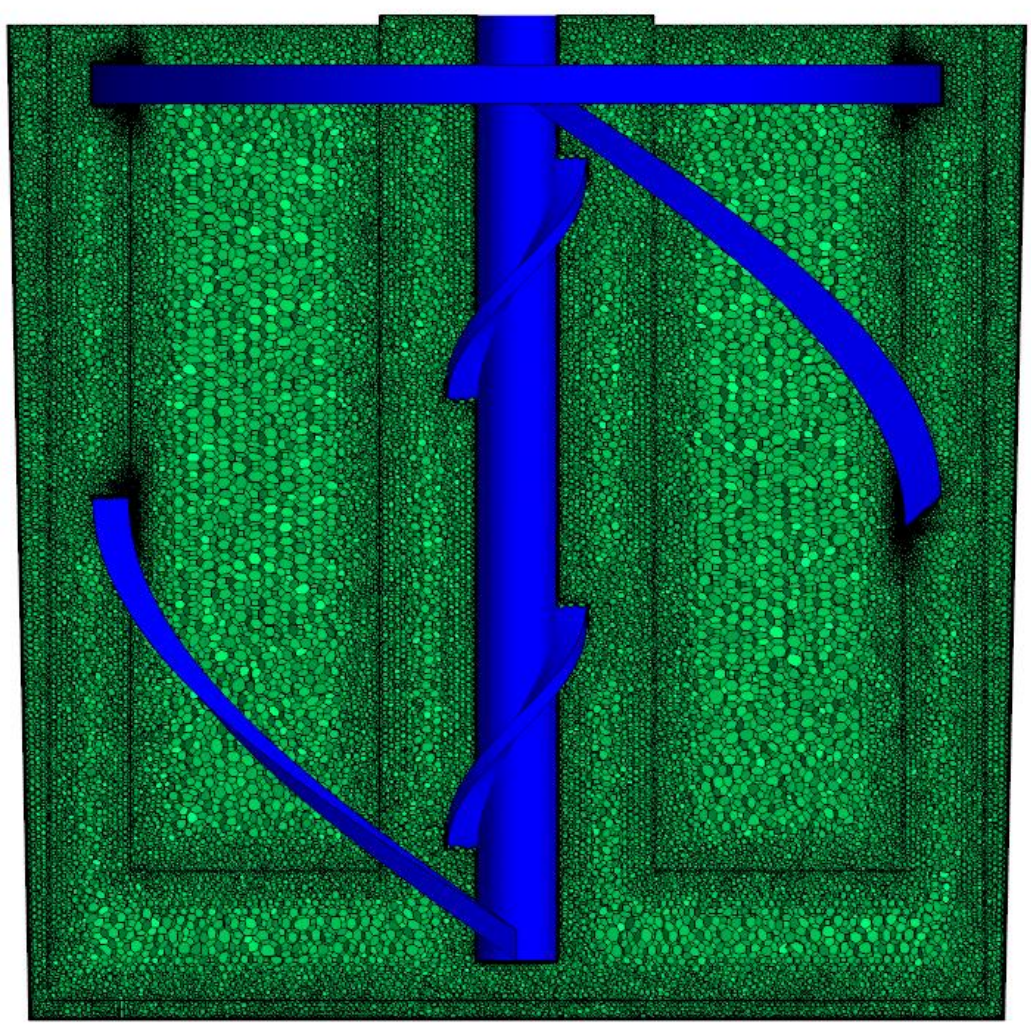

Figure 3. An example of the mesh used to model the torque and mixing response, where it can be seen the finer mesh around the moving mixer elements, i.e. anchor and screw, and coarser mesh in the bulk of the vessel

\subsection{Mixer geometry optimisation}

210 The Siemens HEEDS optimisation package was used to analyse the outputs of the STAR-

$211 \mathrm{CCM}+$ simulations and inform design changes based on the defined constraints. As

212 mentioned previously, the goal was to arrive at the anchor/ribbon design which would

213 maximise the mixing in the system, while keeping the size of the agitator simple, to ease 
cleaning. Therefore, the optimisation package was set to maximise homogeneity and while

215 reducing the torque response, with the schematic of the optimisation loop illustrated in

216 Figure 4. The optimisation process is learning, basing the next design iteration on the

217 outcomes of the previous designs, targeting the desired goal, but limited by the number of

218 iterations predefined by the model set-up, $\mathrm{N}$, where for the purposes of this study $\mathrm{N}=98$

219 (Mariani et al., 2017).

220 It must be noted, that due to the number of design features which are being varied during

221 this study it is possible that more than one design can achieve the desired combination of

222 low torque and high mixing performance.

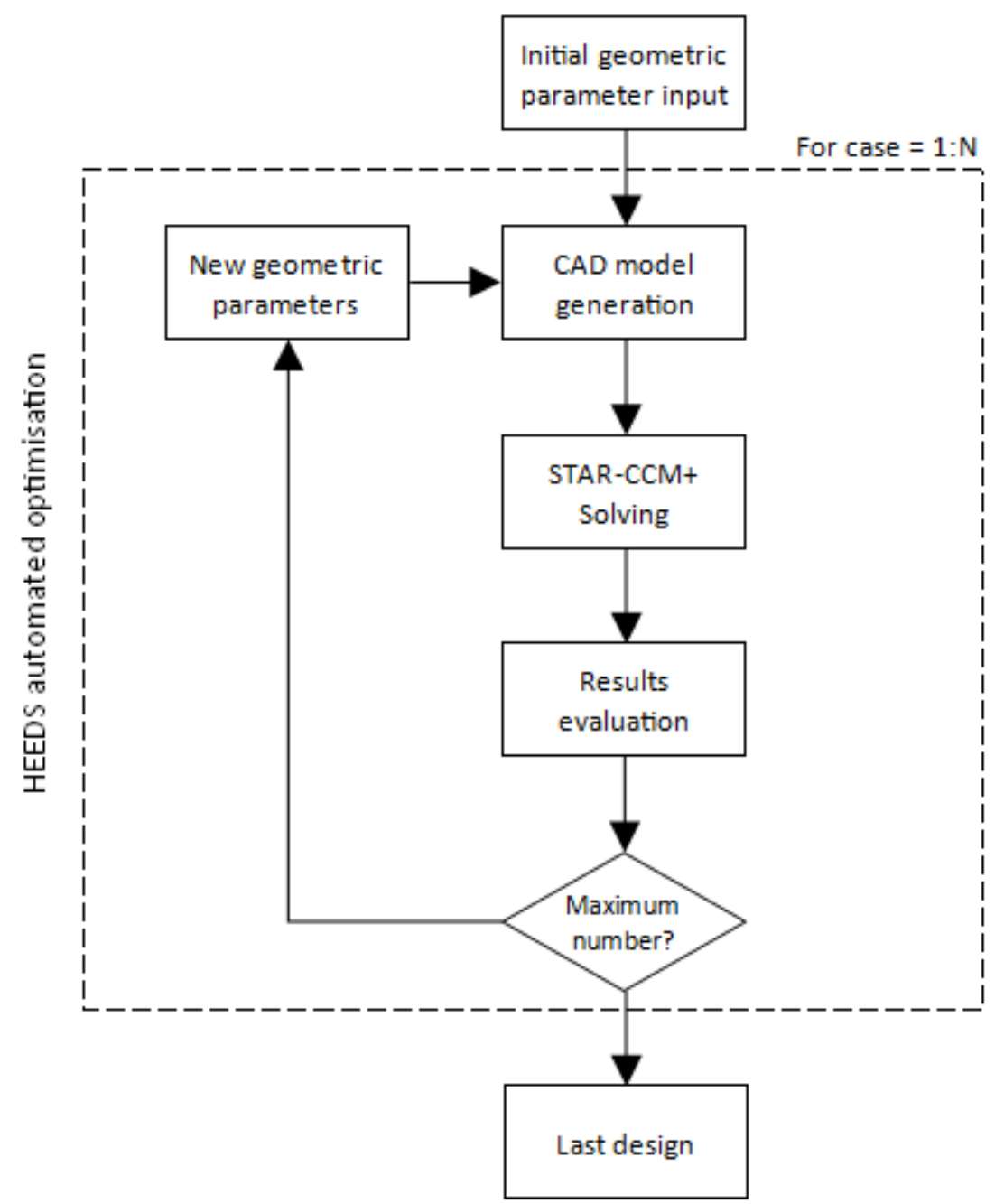




\subsection{Statistical analysis}

226 The CFD output data was fitted to a standard least squares model to investigate the

227 correlation between the mixer performance and the individual features of the mixer design,

228 such as anchor height, number of anchor arms and the number of helix turns. The least

229 squares approach allows to identify outliers and define the strength of the correlation

230 between input and response variables (Robinson and Cleary, 2012).

\subsection{Experimental validation}

\section{2.5.1 Mixer prototype construction for validation}

233 A selection of designs modelled using the STAR-CCM+ and HEEDS packages were chosen for

234 experimental validation. These anchor/ribbon designs were 3D printed using Acrylonitrile

Butadiene Styrene (ABS) plastic through Selective Laser Sintering (SLS), as 3D printing

236 allowed for rapid prototype manufacture in comparison to steel. Furthermore, the use of plastic components allowed the application of Electrical Resistance Tomography (ERT) as one of the validation techniques, whereas the use of a metal mixer elements would interfere with the tracking of the conductivity throughout the system, which the technique

240 relies upon.

241 During the selective laser sintering (SLS) process, small grains of the material are fused

242 together using a laser before a new layer of granular material is deposited on top of the

243 existing structure and process is repeated. SLS is limited to which plastics can be used with

244 the technique, with $A B S$ being one of the most common ones. It is recognised that ABS does

245 not provide the same physical properties as stainless steel which was used for the CFD,

246 however, due to the rigidity of the plastic this was not thought to have had an effect on the

247 mixing performance. The effects of using a different construction material could, 
nevertheless, influence the power draw of the mixer, due to the differences in density and

249 surface roughness between the two materials. Despite these differences, it was concluded

250 that when conducting the torque validation using ABS parts, the trends between the

251 experimental and the CFD values are expected to be consistent, while the actual values may

252 differ due to the construction material.

\section{$253 \quad 2.5 .2$ Torque validation}

254 Using the stand alone mixing module it was possible to carry out the torque validation using 255 a direct measurement obtained from the torque meter (E200 ORT, Sensor Technologies, UK) attached to the anchor motor, capable of measuring the torque response in the range of 0 $200 \mathrm{mN} . \mathrm{m}$ with $0.5 \%$ accuracy, as defined by the manufacturer. The sensor can operate at a higher torque range, of up to $600 \mathrm{mN}$.m, where within this range the accuracy can be reduced, however, this allows to accommodate the wide range of geometry designs, including more complex geometries which result in higher torque response.

For torque validation studies, basic commercially available shampoo formulations were used, with a known viscosity of $10 \mathrm{~Pa} . \mathrm{s}$ at $25{ }^{\circ} \mathrm{C}$, the same as the viscosity of the fluids in the

263 CFD optimisation study. The temperature of the shampoo was maintained at the desired level by immersing the sample pot into a jacketed vessel connected to a temperature controlled bath circulator (Thermo Scientific, UK). Each sample pot was filled with $780 \mathrm{~g}$ of the shampoo, which corresponds to the fill level of $800 \mathrm{ml}$, which is recommended for the

267 mixing module used and is the same as the fill level used in the CFD simulation. To reproduce the conditions of the model, all the elements of the mixer assembly were present in the system during validation (e.g., temperature probe). 
For the torque acquisition step, the mixer assembly was lowered into the pot containing the

271 shampoo and the motor was set to run at $20 \mathrm{rmp}$, the same rotational speed as that used in

272 the CFD. The torque response was recorded every $0.1 \mathrm{~s}$ and each trial ran for 5 minutes.

273 The acquired torque data was analysed, where the first minute of the measurement was

274 discarded, as during this time the readings could be affected by any air trapped in the

275 system when the mixer was lowered in. The remaining data was averaged to provide the

276 value of the torque response of the mixer, the standard deviation of the results was also

277 found, addressing the level of noise in the data.

\section{$278 \quad 2.5 .3 \quad$ Mixing validation}

279 The validation of the mixing performance of the different anchor/ribbon designs was carried

280 out using Electrical Resistance Tomography (ERT), where a conductive tracer, in this case a $25 \%(\mathrm{w} / \mathrm{w}) \mathrm{NaCl}$ solution, was added to the system and the local changes in conductivity indicate the degree of spreading of the tracer and how well it has mixed with the bulk of the system fluid. Tracking these changes is made possible by using the voltage recorded across electrode pairs in the ERT cage, where the voltages can in turn be sued to produce 2- or 3D reconstructions of the conductivity distribution (Polydorides and Lionheart, 2002; Vauhkonen et al., 2001).

287 For the purposes for the validation a bespoke ERT Perspex vessel was manufactured at the 288 University of Manchester which replicates the dimensions of the standard $1 \mathrm{~L}$ sample pots used for the formulation work on the high throughput platform. To enable ERT the

290 technique 6 planes of electrodes, containing 16 electrodes each, were installed in on the walls of the vessel, of which only 5 bottom planes were used, due to the fill level of the vessel. Figure 5 shows the model of the vessel used, where the green rectangles correspond 
294 Systems, UK) the boundary voltages between electrode pairs mounted on the wall of the

295 tank vessel were measured. The change in boundary voltage is related to the change in 296 conductivity in the entire area within the tank vessel, giving localised information during 297 mixing (Yang and Peng, 2003).

298

299

300

301

302

303

304

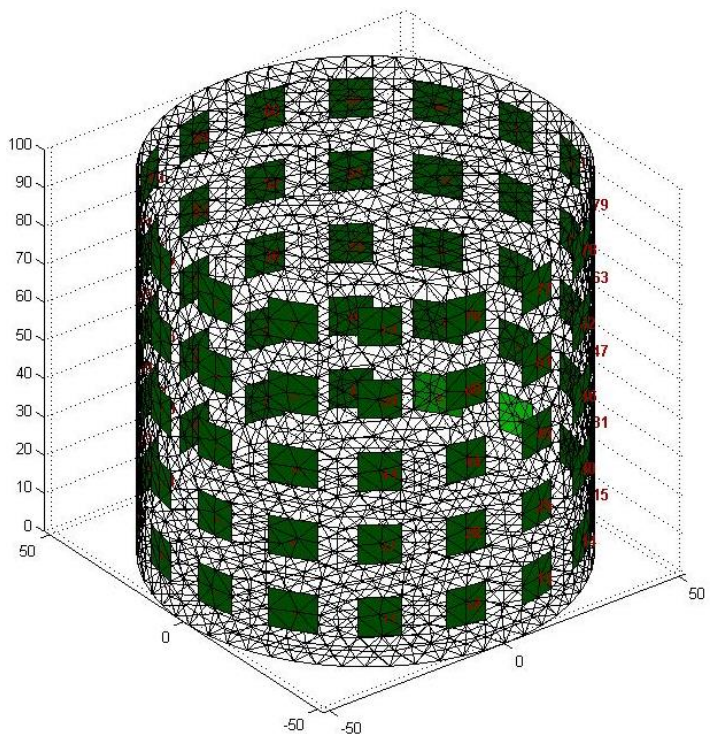

Figure 5. The model of the ERT mixing vessel, where the green rectangles correspond to the positions of the electrodes and the mesh of 14147 finite elements used for conductivity analysis is shown.

The adjacent measurement approach was used, where a fixed current is injected between two adjacent electrodes and voltage is measured across all remaining adjacent electrode pairs, resulting in a total of 520 independent voltage measurements per current injection. The resulting data was processed using the Electrical Impedance Tomography and Diffusion Optical Tomography Reconstruction Software (EIDORS) in MATLAB (Adler and Lionheart, 2006). The sensor model in built in MATLAB was meshed into 14147 finite elements, where the conductivity was calculated for each element based on the 520 voltage readings using the L-curve Tikhonov method (Hansen and O'Leary, 1993). 
The uniformity of the voltage measured between different electrode pairs is then used to

310 estimate how well the high conductivity $\mathrm{NaCl}$ tracer is dispersed across the vessel volume.

\section{Results and Discussions}

\subsection{CFD and mixer geometry optimisation}

313 The results of the optimisation study are summarised in Figure 6, where the torque

314 response of each geometry modelled is presented on the x-axis (N.m), with the

315 homogeneity of the mixture delivered by each design at the end of the simulation is shown

316 on the y-axis. It can be noted that the results are limited by what can be described as a

317 Pareto front, shown in red in the figure, where during the optimisation process no design

318 could be found, within the limits of the defined design features, which would lie outside of

319 this front, hence the Pareto front defines the optimum solution, within the set constraints

320 (Khorram et al., 2014). As described above, the highly desirable features of the mixer for the

321 application on the high throughput formulation platform are high homogeneity at the end

322 of the process, with low torque response. Therefore, any of the designs located towards the

323 top right corner of the Pareto front, before the homogeneity appears to rapidly drop for

324 lower torque designs, would be highly desirable options for the high throughput platform.

325 The designs circled in blue in Figure 6 are shown in detail in Figure 7, with design

326 parameters listed in Table 1, these were chosen for experimental validation purposes. Here

327 some of the designs can be seen to be very similar, for example design 22 and 67 are

328 identical, bar the size of the central Auger screw. Referring to Figure 6 it can be seen that

329 while the larger Auger screw does not appear to provide a significant improvement in the

330 homogeneity of the mixture, it does negatively affect the torque response, adding 
the Auger screw does not enhance the mixing significantly, and when taking into the

333 account the geometry cleaning considerations, should be omitted from the final design. The

334 height of the anchor/helix assembly can be seen to play a significant role in improving the

335 homogeneity of the mixture, as can be seen when comparing the response for designs 67

336 and 81, where full height and 2/3 height mixers are compared, which otherwise have similar

337 features. It can be clearly seen that the mixing performance of the shorter mixer is inferior

338 to that of the full height one. This suggests that when selecting the anchor/helical ribbon

339 design for such applications, the mixer should span as much of the height of the vessel as

340 possible, without introducing other phenomena, such as aeration.

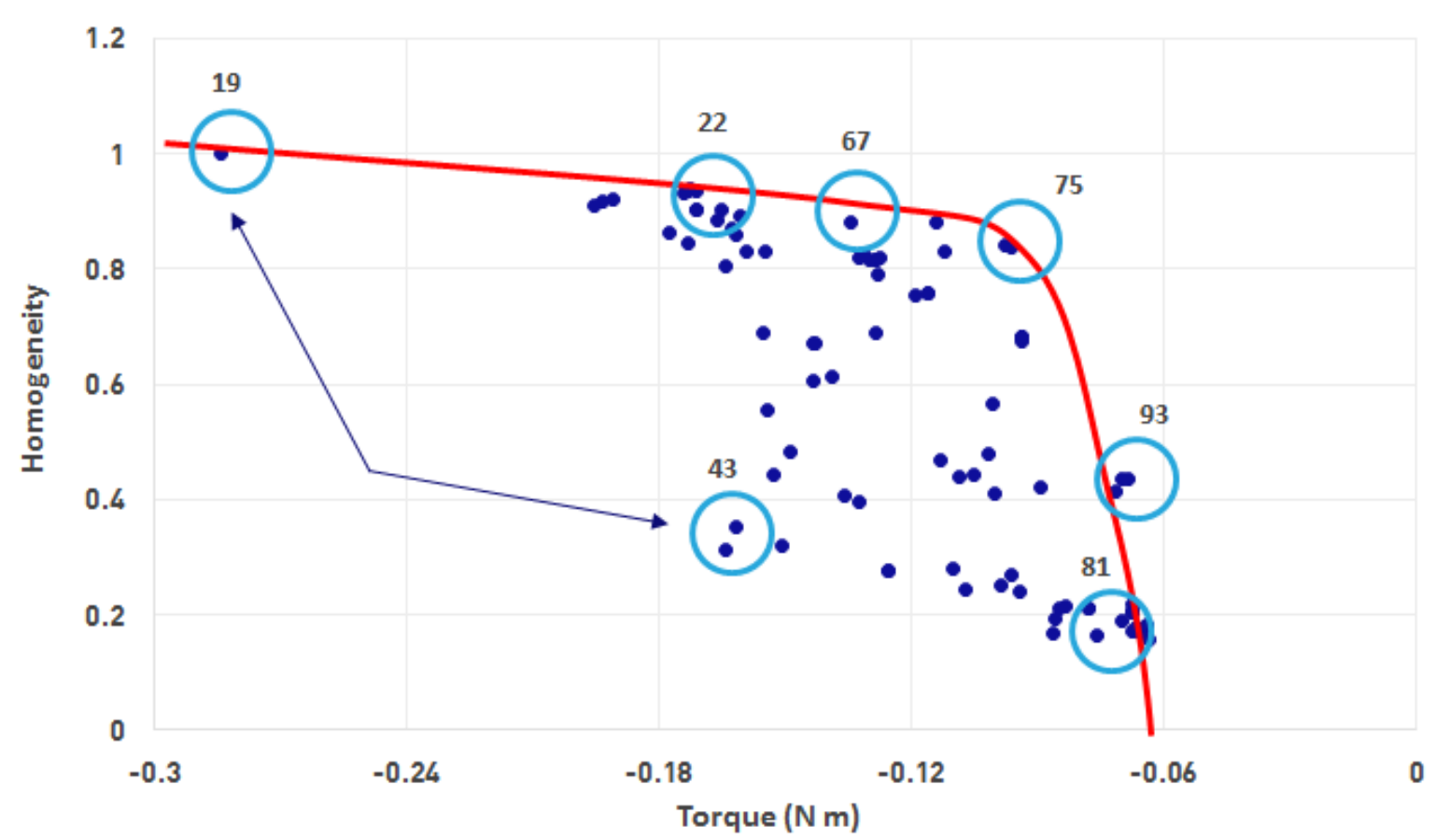

342 Figure 6. Representation of the torque responses and homogeneity of the mixture for all the geometries modelled. The

343 Pareto front is shown in red, while the designs chosen for experimental validation are circled in light blue.

344 It can also be noted that designs 19 and 43 are almost identical, with design 43 having an

345 additional anchor arm, compared to design 19, with no other differences in anchor height, helix or auger screw properties. However, both the homogeneity and the torque response 
347 of the two designs are quite different, which cannot be explained by the geometrical

348 differences between the two designs alone, and can expected to not be consistent with the

349 experimental validation set. Both these designs represent the extremes of the experimental

350 space, as these have most the parameters set to maximum, with the largest Auger screw,

351 the maximum number of helical ribbon elements and the maximum number of turns of the

352 helical ribbon, as well as the maximum number of anchor arms (4) in design 19, and 3 arms

353 in design 43. It can be argued that the model was incapable of accurately calculating the outputs of interest for these designs due to the complexity.

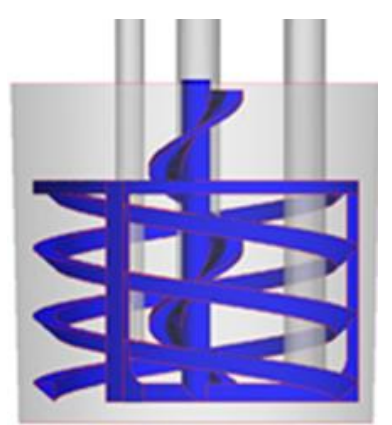

19

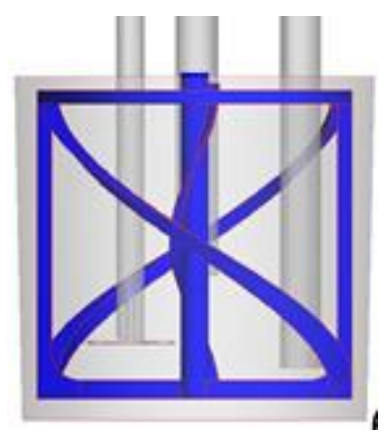

67

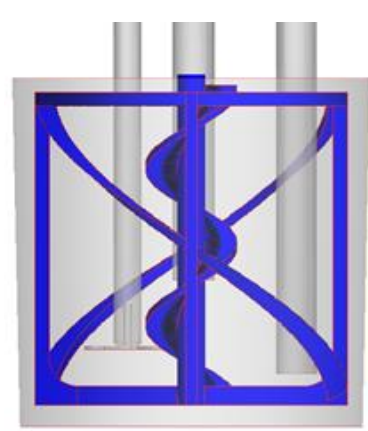

22

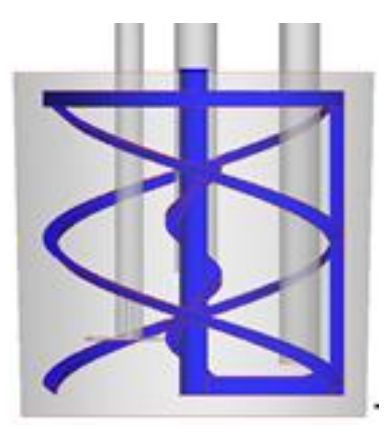

75

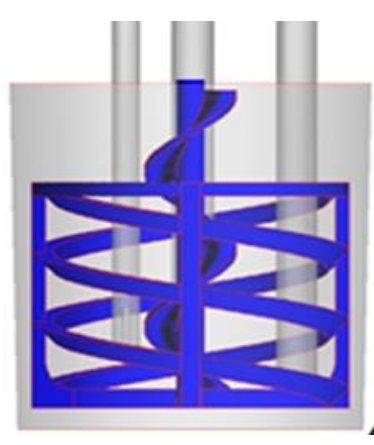

43

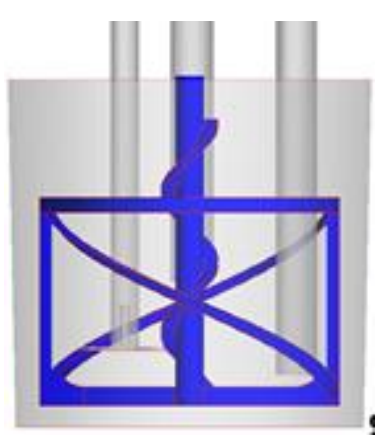

81

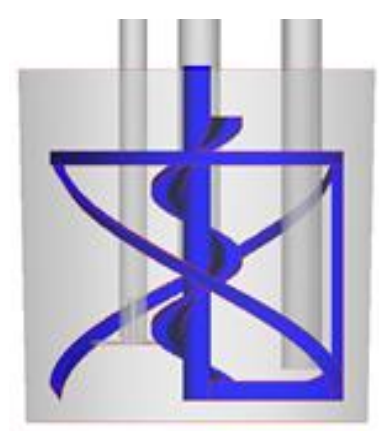


Table 1. Details of geometry designs used for the validation study

\begin{tabular}{|l|l|l|l|l|l|l|l|l|}
\hline $\begin{array}{l}\text { Design } \\
\text { No. }\end{array}$ & $\begin{array}{l}\text { Number } \\
\text { of anchor } \\
\text { arms }\end{array}$ & $\begin{array}{l}\text { Helix } \\
\text { height } \\
(\mathrm{cm})\end{array}$ & $\begin{array}{l}\text { Number } \\
\text { of helix } \\
\text { turns }\end{array}$ & $\begin{array}{l}\text { Helix } \\
\text { pitch } \\
\left({ }^{\circ}\right)\end{array}$ & $\begin{array}{l}\text { Number } \\
\text { of screw } \\
\text { turns }\end{array}$ & $\begin{array}{l}\text { Screw } \\
\text { width } \\
(\mathrm{cm})\end{array}$ & $\begin{array}{l}\text { Area of } \\
\text { anchor } \\
\left(\mathrm{m}^{2}\right)\end{array}$ & $\begin{array}{l}\text { Area of } \\
\text { helix } \\
\left(\mathrm{m}^{2}\right)\end{array}$ \\
\hline 19 & 3 & 6.54 & 1.5 & 15.6 & 2 & 0.9 & $6.4 \mathrm{E}-04$ & $2.1 \mathrm{E}-01$ \\
\hline 22 & 4 & 9.00 & 0.5 & 49.1 & 2 & 0.9 & $1.4 \mathrm{E}-03$ & $4.5 \mathrm{E}-02$ \\
\hline 43 & 4 & 6.54 & 1.5 & 15.6 & 2 & 0.9 & $1.0 \mathrm{E}-03$ & $2.1 \mathrm{E}-01$ \\
\hline 67 & 2 & 9.00 & 0.5 & 49.1 & 1 & 0.3 & $7.2 \mathrm{E}-04$ & $4.5 \mathrm{E}-02$ \\
\hline 75 & 1 & 8.85 & 1 & 29.6 & 2 & 0.4 & $4.0 \mathrm{E}-04$ & $7.9 \mathrm{E}-02$ \\
\hline 81 & 2 & 6.00 & 0.5 & 37.6 & 2 & 0.4 & $5.4 \mathrm{E}-04$ & $3.1 \mathrm{E}-02$ \\
\hline 93 & 1 & 7.08 & 0.5 & 42.2 & 2 & 0.9 & $2.3 \mathrm{E}-04$ & $2.7 \mathrm{E}-02$ \\
\hline
\end{tabular}

359

\subsection{Statistical analysis}

361 While comparing specific features of select designs can provide some information about the

362 effect of the individual design parameters on mixing and torque, systematic statistical

363 analysis can provide quantitative insight into these effects. Therefore, least squares analysis

364 was applied to model the effects of the mixer design parameters on the torque and

365 homogeneity results of the 100 designs modelled, using JMP analytical package (SAS

366 Institute Inc.). Firstly, to test for outliers the residuals of the model were considered, as

367 shown in Figure 8(a), where for homogeneity, any design with a residual of \pm 0.3 was excluded from future analysis (Couturier et al., 2016). For the torque residuals, a similar procedure was performed, leading to excluding two designs, both with the residuals of over 
371 which was suspected to be an outlier based on the inconsistency in results between designs

37243 and 19. A total of 4 outliers were removed using this approach, which were further

373 discovered to have poor correlation with experimental results, during validation.

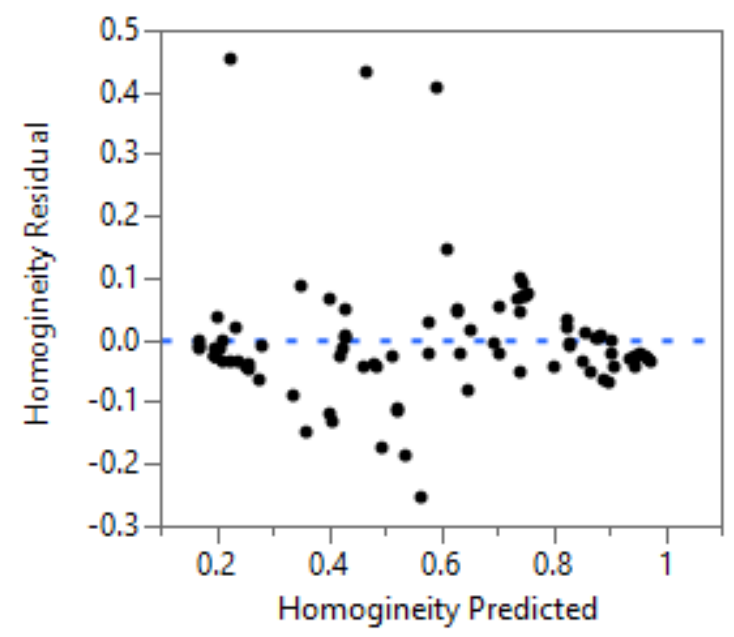

a

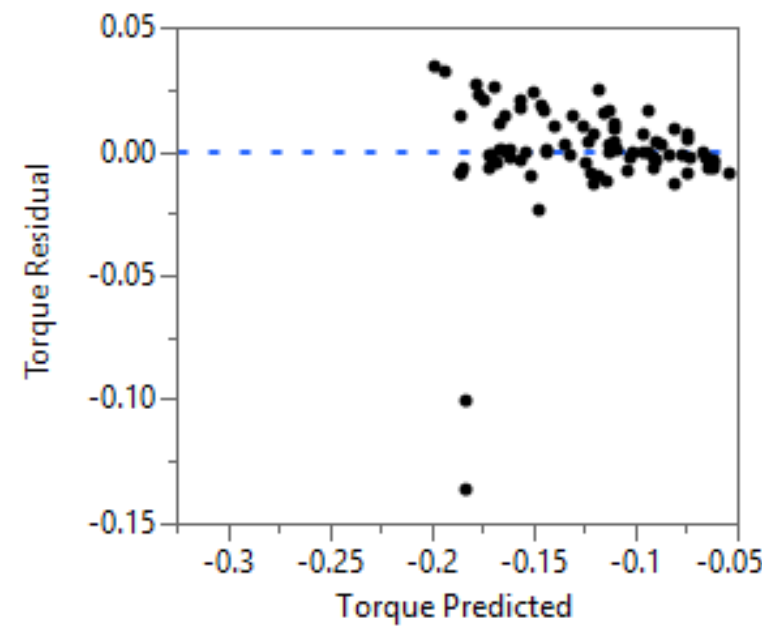

b
374

Figure 8. Residuals from the initial least squares correlation for (a) torque and (b) homogeneity

It can be expected that the torque response of the mixer will be directly correlated with the surface area of the mixer normal to the liquid, i.e. displacing the liquid during motion. This is confirmed by least square analysis where the variables defining torque are the anchor and the helical mixer areas. As can be seen from Figure 9(a) the model based on these variables provides an acceptable fit between actual (CFD) and predicted (least squares) values, with the corresponding $p$-test values for both parameters lying well within the significant range. However, the quality of fit suggests that additional parameters, or level of granularity might be necessary to predict the torque response better. It can be further seen from Figure 9(b) the combination of the two area parameters fails to predict the quality of the mixer performance, i.e. the homogeneity of the mixture at the end of the run. Therefore, further study of the individual design parameters were considered next. 


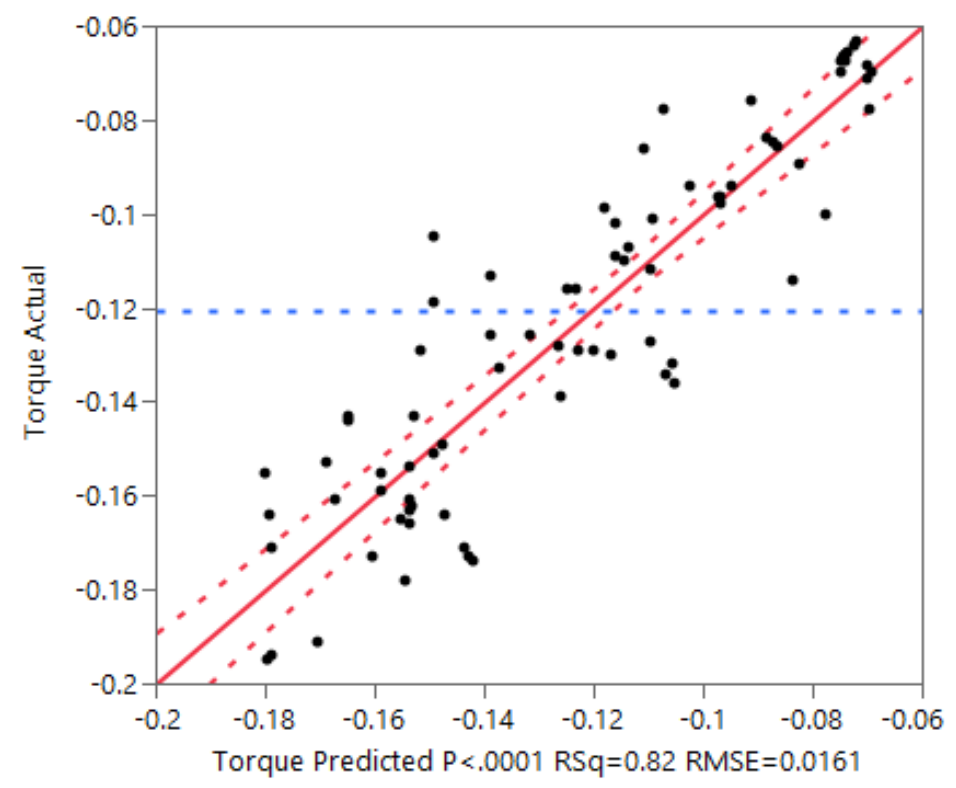

a.

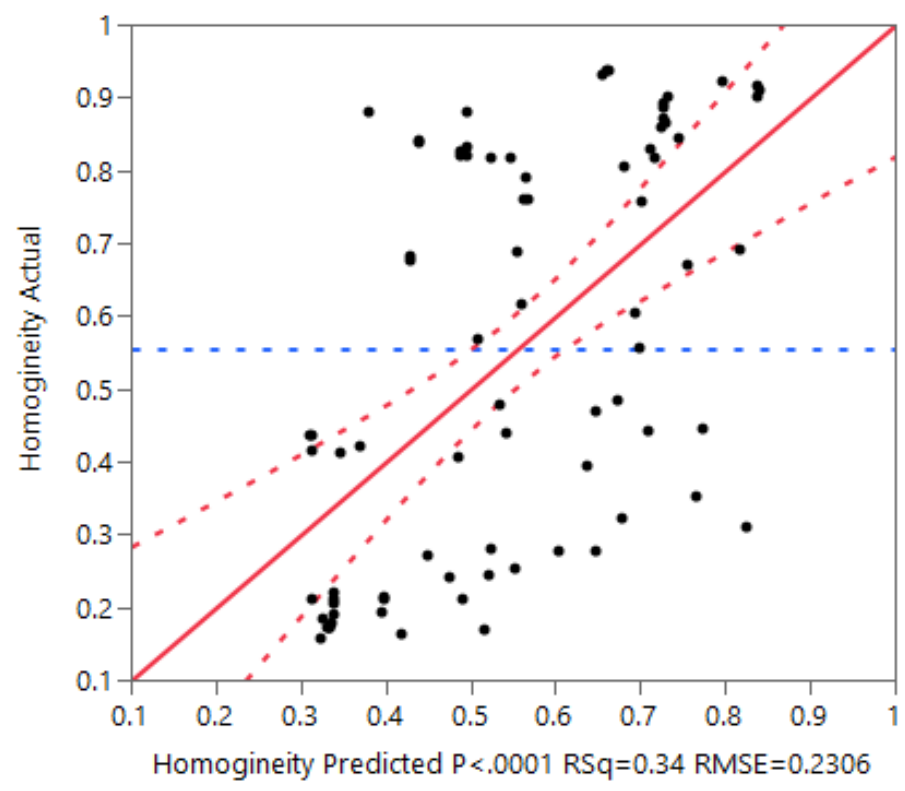

b.

387 Figure 9. Torque (a) and mixing performance (b) least squares models based on the anchor and helical mixer surface areas, where "actual" corresponds to the CFD calculated results, while "predicted" corresponds to the results calculated by the least squares model.

390 Based on the conclusions from the surface area models shown previously, homogeneity and torque models were created using the discrete mixer design features, such as helix height and number of turns, for example. and only direct correlations of parameters considered, 
i.e. no combination effects were included, and neither were non-linear correlations. Figure

39410 illustrates least squares models for the homogeneity (a) and torque response (b), where

395 the $\mathrm{x}$ axis corresponds to the output of the least squares model, while the $\mathrm{y}$ axis corresponds to the results obtained using CFD.

397 Both least squares models provide correlations with high $\mathrm{R}^{2}$ values, 0.97 for both 398 homogeneity and torque, and a low residual mean square error, 0.0545 and 0.0069 respectively, suggesting that not only a strong correlation between the CFD data and the

400 statistical model, but also low deviation from the identity line for individual points on the 401 plot.

402 When looking at the individual effects of the different terms on the expression 403 characterising homogeneity several terms can be identified as statistically significant, as can 404 be seen in Table 2. Based on the p-test values, shown in the $5^{\text {th }}$ column of the table, it can be concluded that the height of the anchor/mixer assembly, width of the helix and the number of helix turns are all significant factors, while the width of the screw, the number of anchor arms and the thickness of the helix are borderline. The coefficient is the highest for

408 the anchor height, suggesting variations that that design parameter would have the greatest effect on the mixing efficiency. The remaining significant terms have relatively small 410 coefficients, suggesting any changes to those design features would not affect the 411 performance of the mixer as much. Therefore, in order to improve mixing the height of the 412 mixer is to be maximised, while the central screw does not provide any mixing benefit. It can 413 also be noted that the width of the anchor does not appear to have a significant impact, and 414 in fact, reducing the number of anchor arms has a positive effect on the mixture homogeneity. This suggests that here the anchor only acts as a supporting structure for the 
416 helical ribbon, but does not significantly contribute to mixing. Based on the above, it can be

417 concluded that the thickness of the anchor as well as the number of arms should be

418 minimised, whenever possible. However, caution is advised, as based on the CFD data the

419 designs providing the best torque/homogeneity outputs could have anchors too thin to

420 ensure structural integrity, or insufficient anchor arms to support the helix effectively, e.g. 1

421 anchor arm in some designs.

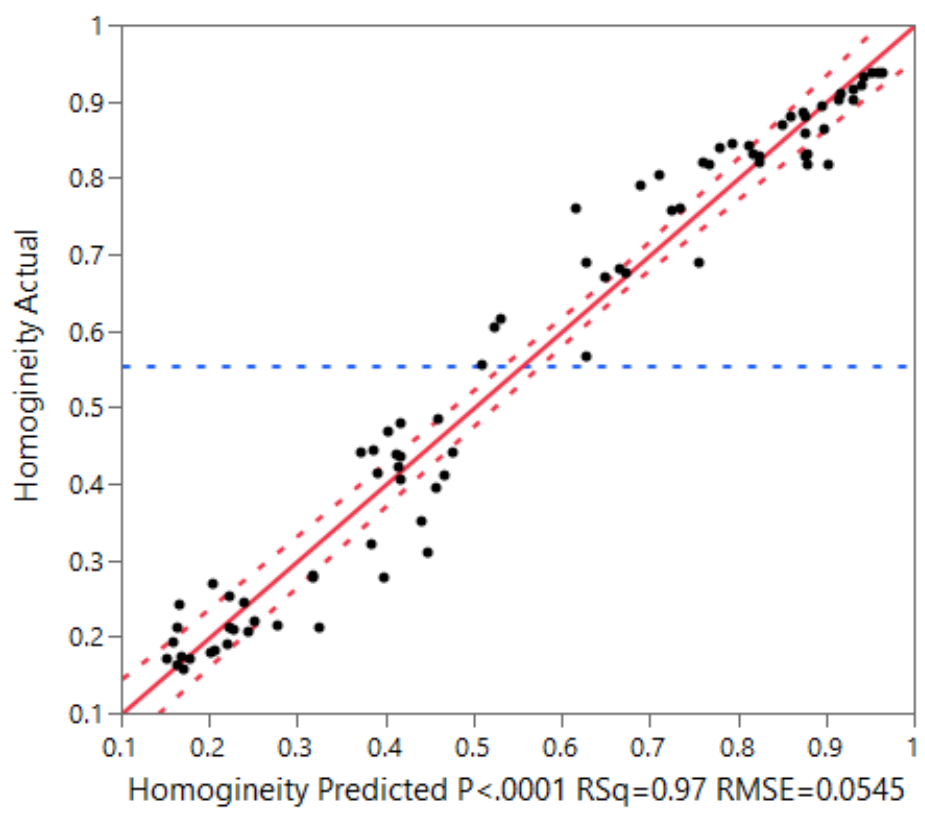

a

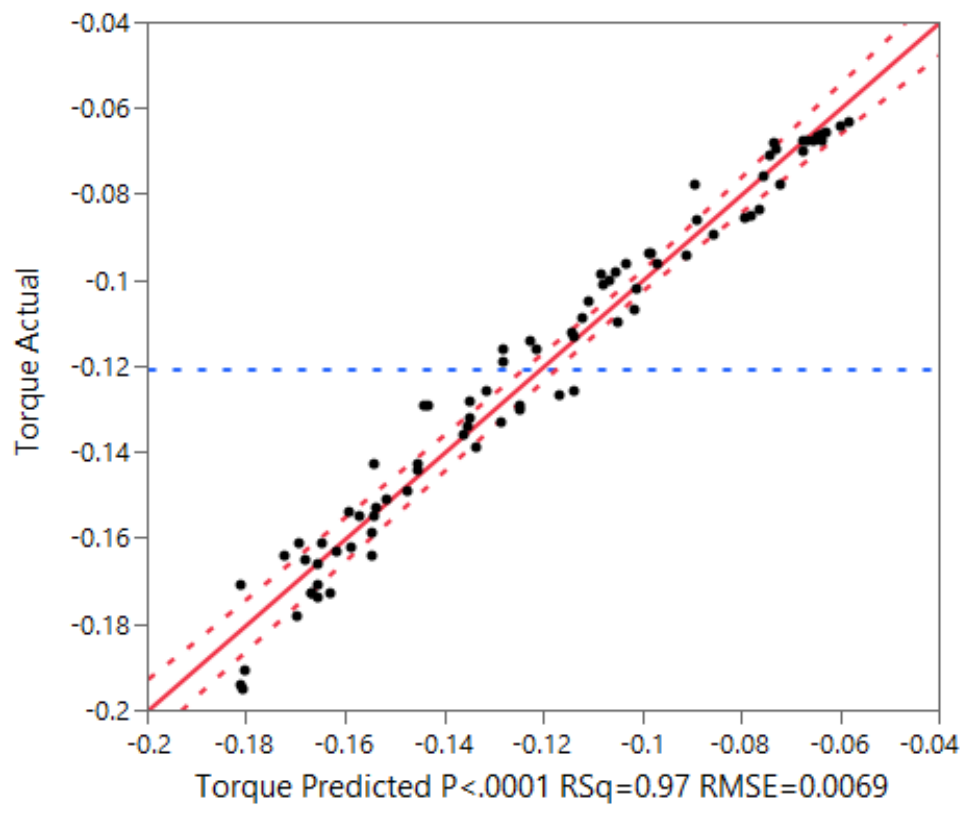


422 Figure 10. Mixing performance (a) and torque (b) least squares models based on individual mixer design features, where

423 "actual" corresponds to the CFD calculated results, while "predicted" corresponds to the results calculated by the least

424 squares model.

425 Table 2. Effect and significance of different design parameters on homogeneity. The effects of parameters marked with (*)

426 are statistically significant at 95\% confidence limit.

\begin{tabular}{l|cccc}
\hline Term & Estimate coefficient & Standard Error & $\mathbf{t}$ Ratio & Probability $>|\mathbf{t}|$ \\
\hline Height of Mixer & 18.7037 & 0.5811 & 32.19 & $<.0001^{*}$ \\
Width of Helix & 0.0767 & 0.0070 & 11.03 & $<.0001^{*}$ \\
No. of helix turns & 0.0550 & 0.0133 & 4.12 & $<.0001^{*}$ \\
Screw width & 0.0098 & 0.0030 & 3.26 & $0.0016^{*}$ \\
No. of anchor arms & -0.0212 & 0.0067 & -3.17 & $0.0022^{*}$ \\
Helix thickness & -0.0258 & 0.0092 & -2.81 & $0.0064^{*}$ \\
Anchor width & -0.0256 & 0.0123 & -2.07 & 0.0416 \\
Screw height & -1.2819 & 0.6609 & -1.94 & 0.0561 \\
No. of screw turns & -0.0237 & 0.0161 & -1.47 & 0.1456 \\
\hline
\end{tabular}

427

428 Similarly, the coefficients defining the significance of the parameters for the torque 429 response are shown in Table 3. Here the significant parameters are the number of anchor 430 arms, the width of the helix, the number of helix turns and the height of the anchor/helix 431 mixer assembly, with the central screw width being borderline and the remaining terms are 432 not significant. This is to be expected, as all of the significant terms can be linked to 433 substantially changing the size of the mixer, and therefore affecting the torque 
measurement. Ideally, to achieve the best design within the desired parameters, the height

435 of the mixer assembly is to be kept at a maximum height which does not introduce aeration,

436 while all the remaining parameters which increase torque should be minimised wherever

437 possible.

Table 3. Effect and significance of different design parameters on torque. The effects of parameters marked with (*) are

\begin{tabular}{l|llll}
\hline Term & Estimate & Standard Error & t Ratio & Probability $>|\mathbf{t}|$ \\
\hline No. of anchor arms & -0.0127 & 0.0009 & -13.93 & $<.0001^{*}$ \\
Width of Helix & -0.0131 & 0.0009 & -13.86 & $<.0001^{*}$ \\
No. of helix turns & -0.0215 & 0.0018 & -11.87 & $<.0001^{*}$ \\
Height of Mixer & -0.8847 & 0.0788 & -11.22 & $<.0001^{*}$ \\
Screw width & -0.0010 & 0.0004 & -2.45 & $0.0165^{*}$ \\
Anchor width & -0.0021 & 0.0017 & -1.34 & 0.1828 \\
Helix thickness & -0.0012 & 0.0012 & -0.93 & 0.3544 \\
No. of screw turns & 0.0015 & 0.0022 & 0.67 & 0.5039 \\
Screw height & -0.0470 & 0.0896 & -0.52 & 0.6015 \\
\hline
\end{tabular}

\section{$441 \quad 3.3 .1$ Torque}

442 For torque validation, the mixer geometries which were 3D printed were attached to the

443 standalone mixer station and set to run at constant rpm, consistent with the rmp of the CFD

444 model, immersed in the silicone oil or shampoo with a defined viscosity, also matching that 445 of the model. 
446 The comparison between the torque response delivered by the model and that obtained

447 experimentally is shown in Figure 11, where 6 out of the 7 designs tested show agreement

448 between the model and experimental data. However, there is consistent offset of 449 approximately $20 \%$ between the two values, with the CFD values higher than the 450 experimental results. This can be attributed to a number of factors, like the construction 451 material of the anchor, where ABS is lighter than steel and also exhibits different surface 452 properties, e.g. roughness.

453 Alternatively, it is possible that the fluid viscosity was not perfectly matched between the 454 model and the experiment, either due to error in off-line viscosity measurements or 455 temperature control during the experiment.

456 Nevertheless, this error appears to be systematic, and does not prevent from concluding 457 that trend in the torque response based on CFD is consistent with experimental 458 measurements.

459 Geometry 19 must also be highlighted as an outlier, this was expected based on the 460 observations of the Pareto front results and the least squares model used to determine the 461 significant design parameters. Here, geometries 19 and 43 are known to be very 462 geometrically similar, and are expected to provide similar torque response, which can be 463 seen from the experimental results, with the recorded torque of 0.1297 and 0.1254 N.m 464 respectively for the two geometries. However, the CFD results show a dramatic difference, 465 with geometry 19 yielding a torque response of $0.320 \mathrm{~N} . \mathrm{m}$, almost double of the $0.164 \mathrm{~N} . \mathrm{m}$

466 for geometry 43 . It is reasonable to conclude that the value obtained for geometry 43 is the 467 correct one, as it falls in line with the results for other geometries used in the validation 

the mistake within the software when setting up the CFD parameters.

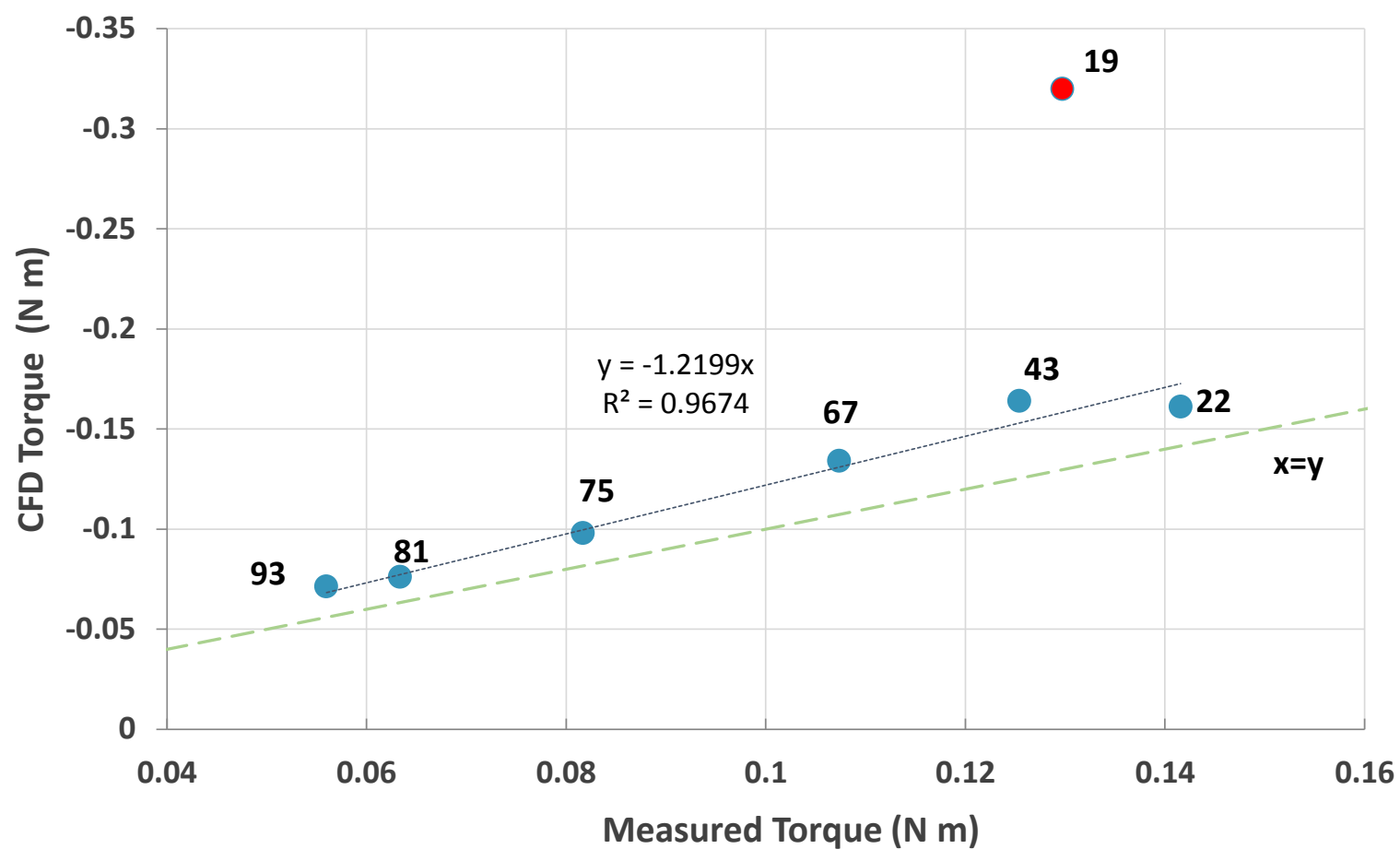

470

471 Figure 11. The comparison between the experimentally measured torque and torque calculated using CFD. Here the

472 individual points represent the different geometry designs, with a linear trendline showing the correlation between the 473 experimentally measured torque ( $x$ axis) and that calculated using CFD (y axis), with the CFD results shown to exceed the 474 experimental results by approximately 20\%. Geometry 19 is shown to be an outlier and is marked in red. A dashed $x=y$ line is 475 added for reference, representing a theoretical perfect agreement between experimental and CFD results.

\subsubsection{Mixing}

477 Mixing was validated using ERT, where a conductive tracer was added to the bulk of less conductive liquid and the conductivity across the volume of the vessel was recorded over

479 time, until homogeneity was reached. As the homogeneity of the mixture in the CFD predicted results was measured at the end of the simulation, as opposed to continuously over time, it cannot be directly compared with the mixing times required to achieve a fully mixed system, which is calculated using ERT. However, it is still possible to draw parallels 
483 between the homogeneity and mixing time, as shown in Figure 12. Here, for mixer designs

484 which have achieved high homogeneity at the end of the CFD simulation maintain a plateau

485 at low mixing times, as no differentiation can be made between designs which completed

486 the mixing process at the end of the simulation run. However, as the CFD predicted

487 homogeneity at the end of simulation allotted time declines the experimental time required

488 to achieve a fully homogeneous mixture can be observed to increase, illustrating that it

489 takes longer to achieve a homogeneous mixture for the mixer designs which perform

490 poorly.

491 Here design 43 appears to be an outlier, as the actual mixing time required to achieve a fully

492 mixed system using this design is lower than that predicted using the model, however, this

493 design was excluded from the least squares model based on the model residuals, and is

494 therefore expected to perform out of line with the other designs. 


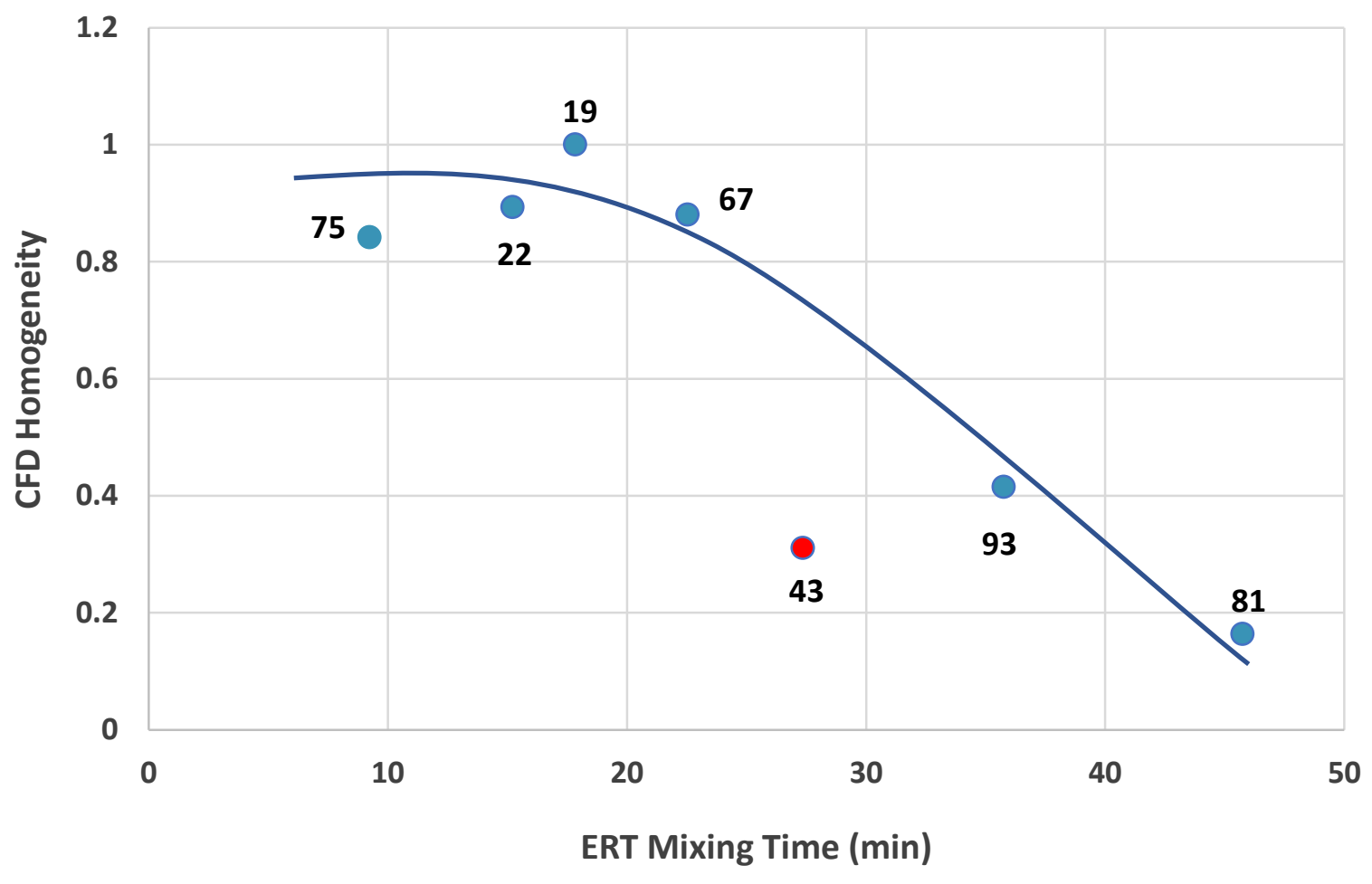

496 Figure 12. the comparison between the experimentally measured mixing time and the homogineity of the fluid in the vessel

497 at the end of the CFD simulation run.

\section{Conclusions}

499 STAR-CCM+ CFD package and HEEDS optimisation package to define the optimal design for a

500 small scale $1 \mathrm{~L}$ mixer anchor mixer. Over 100 designs were processed by the CFD package changing a number of geometrical parameters, such as the height of the anchor/helix mixer assembly, the number of anchor arms and the number of turns of the helix, as well as the

503 effect of the central Auger screw. The model provided outputs on the torque response and 504 the mixing performance of each design, which were validated experimentally, using a stand505 alone module of a high throughput platform formulator, equipped with a torque sensor and an ERT conductivity measurement system.

507 The outputs of the model were analysed by fitting the data to a standard least squares 508 model, identifying several outliers and defining the key characteristics of the mixer designs 
which affect mixing and torque. It is desirable to reduce torque while increasing mixing

510 efficiency, both from a power efficiency perspective and in order to ensure ease of cleaning.

511 It was found that the height of the mixer assembly is the key driver to the mixers

512 performance, however, the anchor itself does not contribute to improving mixing, with the

513 helical ribbon features, such as width and number of turns playing a significant role in

514 achieving a highly homogeneous mixture. In addition, it was determined that the central

515 Auger screw does not have a statistically significant effect on mixing.

516 In turn, torque was determined to be highly dependent on the height of the mixer assembly,

517 the number of mixer arms, the number of helix turns and the width of the helix, where all

518 these parameters also significantly influence the size of the mixer, which in turns is directly

519 linked to increased torque in real systems.

520 It is can therefore be advised to maximise the height of the mixer whenever practical, while 521 reducing the size of the other design features.

522 The use of CFD for the estimation of the effect of different mixer design features on the two

523 key parameters, torque and mixing performance, has been shown to be an effective

524 approach for rapid optimisation of a mixer design for a given set of constraints.

\section{Acknowledgements}

526 The authors would like to thank InnovateUK for the financial support provided for this work

527 as a part of the Embedding Manufacturing Development into Formulation Research 528 (EMFormR) project (EP/L505778/1). The authors would also like to thank the Unilever Port 529 Sunlight R\&D rapid prototyping team for help with 3D printing of mixer parts. 
531 Adler, A., Lionheart, W.R.B., 2006. Uses and abuses of EIDORS: an extensible software base

532 for EIT. Physiol. Meas. 27, S25-S42. doi:10.1088/0967-3334/27/5/S03

Alberini, F., Liu, L., Stitt, E.H., Simmons, M.J.H., 2017. Comparison between 3-D-PTV and 2-DPIV for determination of hydrodynamics of complex fluids in a stirred vessel. Chem. Eng. Sci. 171, 189-203. doi:10.1016/j.ces.2017.05.034

Ameur, H., 2016. Effect of some parameters on the performance of anchor impellers for stirring shear-thinning fluids in a cylindrical vessel. J. Hydrodyn. 28, 669-675. doi:10.1016/S1001-6058(16)60671-6

Ayazi Shamlou, P., Edwards, M.F., 1985. Power consumption of helical ribbon mixers in viscous newtonian and non-newtonian fluids. Chem. Eng. Sci. 40, 1773-1781. doi:10.1016/0009-2509(85)80040-3

Balzer, D., Varwig, S., Weihrauch, M., Ag, H., Marl, D.-, 1995. Viscoelasticity of personal care products. Colloids Surfaces A 99, 233-246. doi:10.1016/0927-7757(95)03144-3

544 Brito-De La Fuente, E., Choplin, L., Tanguy, P., 1997. Mixing With Helical Ribbon Impellers. Chem. Eng. Res. Des. 75, 45-52. doi:10.1205/026387697523381

546 Chua, C.K., Wong, C.H., Yeong, W.Y., n.d. Standards, quality control, and measurement sciences in 3D printing and additive manufacturing.

548 Couturier, R., Ziane Khodja, L., Guyeux, C., 2016. TSIRM: A two-stage iteration with leastsquares residual minimization algorithm to solve large sparse linear and nonlinear 
551

552

553

554

555

556

557

558

559

560

561

562

563

564

565

566

567

568

569

570

571

Doraiswamy, D., Grenville, R.K., Etchells, A.W., 1994. Two-Score Years of the Metzner-Otto Correlation. Ind. Eng. Chem. Res. 33, 2253-2258. doi:10.1021/ie00034a001

Ferziger, J.H., Perić, M., 2002. Computational Methods for Fluid Dynamics, 3rd ed. Springer Berlin Heidelberg, Berlin, Heidelberg. doi:10.1007/978-3-642-56026-2

Gijón-Arreortúa, I., Tecante, A., 2015. Mixing time and power consumption during blending of cohesive food powders with a horizontal helical double-ribbon impeller. J. Food Eng. 149, 144-152. doi:10.1016/j.jfoodeng.2014.10.013

Han, Y., Wang, J.J., Gu, X.P., Feng, L.F., 2012. Numerical simulation on micromixing of viscous fluids in a stirred-tank reactor. Chem. Eng. Sci. 74, 9-17. doi:10.1016/j.ces.2012.02.018

Hanada, T., Kuroda, K., Takahashi, K., 2016. CFD geometrical optimization to improve mixing performance of axial mixer. Chem. Eng. Sci. 144, 144-152. doi:10.1016/j.ces.2016.01.029

Hansen, P.C., O'Leary, D.P., 1993. The Use of the L-Curve in the Regularization of Discrete IIIPosed Problems. SIAM J. Sci. Comput. 14, 1487-1503. doi:10.1137/0914086

Khorram, E., Khaledian, K., Khaledyan, M., 2014. A numerical method for constructing the Pareto front of multi-objective optimization problems. J. Comput. Appl. Math. 261, 158-171. doi:10.1016/j.cam.2013.11.007

Kuhs, M., Moore, J., Kollamaram, G., Walker, G., Croker, D., 2017. Predicting optimal wet granulation parameters for extrusion-spheronisation of pharmaceutical pellets using a mixer torque rheometer. Int. J. Pharm. 517, 19-24. doi:10.1016/j.ijpharm.2016.11.057 
572

573

574

575

576

577

578

579

580

581

582

583

584

585

586

587

588

589

590

591

592

Mariani, F., Risi, F., Grimaldi, C.N., 2017. Separation efficiency and heat exchange optimization in a cyclone. Sep. Purif. Technol. 179, 393-402. doi:10.1016/j.seppur.2017.02.024

McCarthy, K.L., Lee, Y., Green, J., McCarthy, M.J., 2002. Magnetic resonance imaging as a sensor system for multiphase mixing. Appl. Magn. Reson. 22, 213-222.

Mihailova, O., Lim, V., McCarthy, M.J., McCarthy, K.L., Bakalis, S., 2015. Laminar mixing in a SMX static mixer evaluated by positron emission particle tracking (PEPT) and magnetic resonance imaging (MRI). Chem. Eng. Sci. 137, 1014-1023.

doi:10.1016/j.ces.2015.07.015

Palacz, M., Smolka, J., Kus, W., Fic, A., Bulinski, Z., Nowak, A.J., Banasiak, K., Hafner, A., 2016. CFD-based shape optimisation of a CO2 two-phase ejector mixing section. Appl. Therm. Eng. 95, 62-69. doi:10.1016/j.applthermaleng.2015.11.012

Polydorides, N., Lionheart, W.R.B., 2002. A Matlab toolkit for three-dimensional electrical impedance tomography: a contribution to the Electrical Impedance and Diffuse Optical Reconstruction Software project. Meas. Sci. Technol. 13, 1871-1883. doi:10.1088/0957-0233/13/12/310

Ramsay, J., Simmons, M.J.H., Ingram, A., Stitt, E.H., 2016. Mixing performance of viscoelastic fluids in a Kenics KM in-line static mixer. Chem. Eng. Res. Des. 115, 310-324. doi:10.1016/j.cherd.2016.07.020

Robinson, M., Cleary, P.W., 2012. Flow and mixing performance in helical ribbon mixers. Chem. Eng. Sci. 84, 382-398. doi:10.1016/j.ces.2012.08.044 
593

594

595

596

597

598

599

600

601

602

603

604

605

606

607

Rodgers, T.L., Kowalski, A., 2010. An electrical resistance tomography method for determining mixing in batch addition with a level change. Chem. Eng. Res. Des. 88, 204-212. doi:10.1016/j.cherd.2009.08.003

Vauhkonen, M., Lionheart, W.R.B., Heikkinen, L.M., Vauhkonen, P.J., Kaipio, J.P., 2001. A MATLAB package for the EIDORS project to reconstruct two-dimensional EIT images. Physiol. Meas. 22, 107-111. doi:10.1088/0967-3334/22/1/314

Vicum, L., Ottiger, S., Mazzotti, M., Makowski, Ł., Bałdyga, J., 2004. Multi-scale modeling of a reactive mixing process in a semibatch stirred tank. Chem. Eng. Sci. 59, 1767-1781. doi:10.1016/j.ces.2004.01.032

Yang, W.Q., Peng, L., 2003. Image reconstruction algorithms for electrical capacitance tomography. Meas. Sci. Technol. 14, R1-R13. doi:10.1088/0957-0233/14/1/201

Zhang, M., Zhang, L., Jiang, B., Yin, Y., Li, X., 2008. Calculation of Metzner Constant for Double Helical Ribbon Impeller by Computational Fluid Dynamic Method. Chinese J. Chem. Eng. 16, 686-692. doi:10.1016/S1004-9541(08)60141-X 